$>$ L'individualisation du traitement immunosuppresseur fait intervenir le suivi thérapeutique pharmacologique (STP) par la détermination du meilleur point unique ou la réalisation d'aires sous la courbe abrégées (abbreviated area under the curve-AUC), établies à partir des concentrations sanguines d'un médicament. Des travaux récents ont montré que la concentration de ciclosporine deux heures après la prise était le meilleur point unique. Cependant, une analyse critique laisse planer un doute sur l'intérêt clinique de cette approche. Les cibles de l'AUC de l'acide mycophénolique sont comprises entre 30 et $60 \mathrm{mg}$.h/l, mais les résultats cliniques discordants de deux études randomisées ne permettent pas de donner des conclusions claires sur son intérêt clinique. La pharmacogénétique est l'étude de l'influence de polymorphismes génétiques sur la réponse individuelle à un traitement. II peut s'agir d'une réponse pharmacocinétique ou pharmacodynamique en terme d'efficacité ou de tolérance. Pour le tacrolimus, les posologies nécessaires pour obtenir les concentrations souhaitées sont corrélées à l'expression du cytochrome P450 3A5 (allèle *1). Une étude prospective de pharmacogénétique est en cours pour démontrer l'utilité de cette approche. «

\section{Apports récents de la pharmacologie des traitements immunosuppresseurs utilisés en transplantation d'organe}

\section{Eric Thervet}

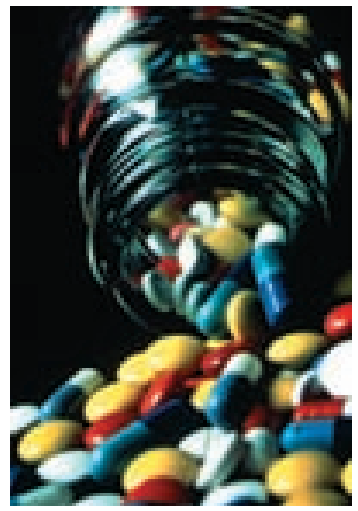

Service de Transplantation et de Soins intensifs, Hôpital Necker, 149 , rue de Sèvres, 75015 Paris, France. eric.thervet@nck.aphp.fr
La transplantation rénale est le traitement de choix de l'insuffisance rénale chronique au stade terminal. Les progrès récents sont dus à la diminution de l'incidence des rejets aigus, témoin de l'efficacité de l'immunosuppression, et à une meilleure prévention des complications infectieuses. Pour mieux équilibrer cette balance risque/bénéfice, l'individualisation de l'immunosuppression est l'une des priorités avec l'utilisation de nouveaux outils pharmacocinétiques et pharmacogénétiques.

La pharmacocinétique classique a laissé la place à la notion de suivi thérapeutique pharmacologique (STP) qui permet, par le dosage des concentrations sanguines d'un médicament, d'ajuster la dose adminis- trée à un individu directement ou à l'aide de modèles pharmacocinétiques. Pour qu'un médicament soit éligible au STP, sa réponse pharmacologique doit être difficilement accessible par une mesure d'effet (un contre-exemple étant les anti-hypertenseurs et la mesure de la pression artérielle), son effet doit être en rapport plus étroit avec les concentrations obtenues dans le compartiment sanguin qu'avec la posologie quotidienne prise, il doit présenter une zone thérapeutique étroite et il doit exister une grande variabilité pharmacocinétique inter- ou intra-individuelle. C'est le cas des traitements immunosuppresseurs. Dans cet article, nous discuterons de l'intérêt du STP appliqué aux inhibiteurs de l'inosine monophosphate déshydrogénase (IMPDH) et de la pertinence des outils pharmacocinétiques choisis pour la ciclosporine (CsA).

La pharmacogénétique, quant à elle, est l'étude de l'influence des polymorphismes génétiques d'enzymes du métabolisme des xénobiotiques et de transporteurs sur la pharmacocinétique ou la réponse clinique de certains traitements. Nous analyserons ici les récentes avancées dans ce domaine portant sur l'utilisation du tacrolimus (TAC) et du sirolimus (SRL). 


\section{Exemple 1 : la ciclosporine}

Dès le début de son utilisation, il est apparu que la CsA présente un index thérapeutique étroit et que son rapport risque/bénéfice peut être significativement amélioré par l'utilisation d'un STP [1]. Des concentrations sanguines basses de CsA sont associées à un risque augmenté

\begin{tabular}{|c|c|c|}
\hline Équipes & $\begin{array}{c}\text { Régime } \\
\text { immunosuppresseur }\end{array}$ & C2 cibles $(\mathrm{ng} / \mathrm{ml})$ \\
\hline \multicolumn{3}{|l|}{ Transplantation rénale } \\
\hline Anvers (Belgique) & CsA, MPA, STE \pm antiCD25 & $\begin{array}{c}\text { M1: } 1300-1500 \\
M 2-3: 900-1300 \\
M 4-6: 750-950 \\
\quad>\text { M6: } 700\end{array}$ \\
\hline Oslo (Norvège) & CsA, MPA, STE & $\begin{array}{l}M 1: 1500-2000 \\
M 2: 1400-1600 \\
M 3: 1000-1200\end{array}$ \\
\hline \multirow[t]{3}{*}{ Berlin (Allemagne) } & CsA, MPA, STE & $\begin{array}{l}M 1: 1400-1600 \\
M 2-6: 800-1100 \\
>\text { M6: } 450-700\end{array}$ \\
\hline & CsA, MPA, STE, AntiCD25 & $\begin{array}{l}M 1: 1000-1100 \\
M 2-6: 700-900 \\
>M 6: 450-700\end{array}$ \\
\hline & CsA, Inh mTOR, STE & $\begin{array}{c}M 1: 500-700 \\
M 2-6: 400-600 \\
M 7-12: 300-500 \\
>\text { M12 : 200-400 }\end{array}$ \\
\hline Newcastle (Angleterre) & CsA, AZA, STE, AntiCD25 & $\begin{array}{l}M 1-3: 1000-1500 \\
M 4-6: 800-1200 \\
>M 6: 600-1000\end{array}$ \\
\hline Paris Necker (France) & CsA, MPA, STE, AntiCD25 & $\begin{array}{c}M 1-3: 1000-1200 \\
M 4-6: 800-1000 \\
>M 6: 600-800\end{array}$ \\
\hline \multicolumn{3}{|c|}{ Transplantation hépatique } \\
\hline \multirow[t]{2}{*}{$\begin{array}{l}\text { Corufia (Espagne) } \\
\text { Hanovre (Allemagne) }\end{array}$} & CsA, MPA, STE & $\begin{array}{l}M 1: 800-1000 \\
M 2-6: 600-800 \\
\quad>M 6: 600\end{array}$ \\
\hline & CsA, MPA, anti-CD25 & $\begin{array}{c}\mathrm{Ml}: 600-800 \\
>\mathrm{Ml}: 600\end{array}$ \\
\hline Caen (France) & CsA, MPA, STE & $\begin{array}{c}M 1: 800-1200 \\
M 2-3: 800-1000 \\
M 4-6: 600-800 \\
>M 6: 400-600\end{array}$ \\
\hline Turin (Italie) & CsA, MPA, STE & $\begin{array}{l}M 1: 800-1000 \\
M 2: 600-800 \\
>M 6: 400-600\end{array}$ \\
\hline
\end{tabular}

de rejet et de perte de greffon [2]. Même si les concentrations résiduelles ( $\mathrm{CO}$ ) n'ont qu'une mauvaise valeur prédictive individuelle [3], elles sont devenues le standard du suivi en raison de la facilité de leur réalisation pratique en clinique humaine puisque les patients viennent dans ce cas réaliser leurs examens sanguins à jeun et avant toute prise médicamenteuse. Lors de l'introduction d'une nouvelle forme galénique de CsA (microémulsion ou Néoral ${ }^{\circledR}$ ), des profils pharmacocinétiques complets ont été réalisés $[4,5]$. Ces études ont démontré que l'exposition à la CsA pendant les 4 premières heures suivant l'administration (aire sous la courbe de 0 à 4 heures ou $A C_{0-4}$ ) était corrélée à I'AUC durant l'intervalle complet entre deux prises $\left(A \cup C_{0-12}\right)$ puisque la période entre 0 et 4 heures est celle au cours de laquelle existe la plus grande variabilité de la concentration sanguine de CsA (Figure I). Une étude rétrospective a montré que les patients présentant un épisode de rejet avaient une $\mathrm{AUC}_{0-4}$ basse alors que les patients présentant une néphrotoxicité avaient une $\mathrm{AUC}_{0-4}$ haute et que les valeurs optimales durant les premiers jours suivant la transplantation étaient comprises entre 4400 et $5500 \mu \mathrm{g} / \mathrm{h} / \mathrm{I}$ [6]. Ces valeurs ont été validées secondairement lors d'études prospectives pour des patients avec ou sans traitement d'induction biologique. L'utilisation de ces AUC courtes n'est pas toujours facile en clinique. La concentration mesurée deux heures après la prise $\left(C_{2}\right)$ a été choisie car elle présente la meilleure corrélation avec I'AUC ${ }_{0-4}$ [4] en transplantation rénale [7], hépatique [8], cardiaque [9] et pulmonaire [10].

Le groupe CONCERT a publié la première conférence de consensus portant sur l'utilisation de la C2 [11]. Le prélèvement sanguin doit être réalisé dans une fenêtre de 15 minutes autour de la deuxième heure. L'analyse des données cliniques a montré qu'il existe une association entre les valeurs de C2 et le risque de survenue d'un épisode de rejet après transplantation

Tableau I. Recommandations pour les concentrations de C2 (concentration mesurée 2 heures après la prise) cibles après transplantation. CSA : ciclosporine microémulsion; MPA: inhibiteur de I'IMPDH; STE : stéroïdes; AntiCD25 : anticorps monoclonal anti-récepteur de l'interleukine 2; AZA: azathioprine; Inh mTOR : inhibiteurs de la mammalian target of rapamycine [12]. 


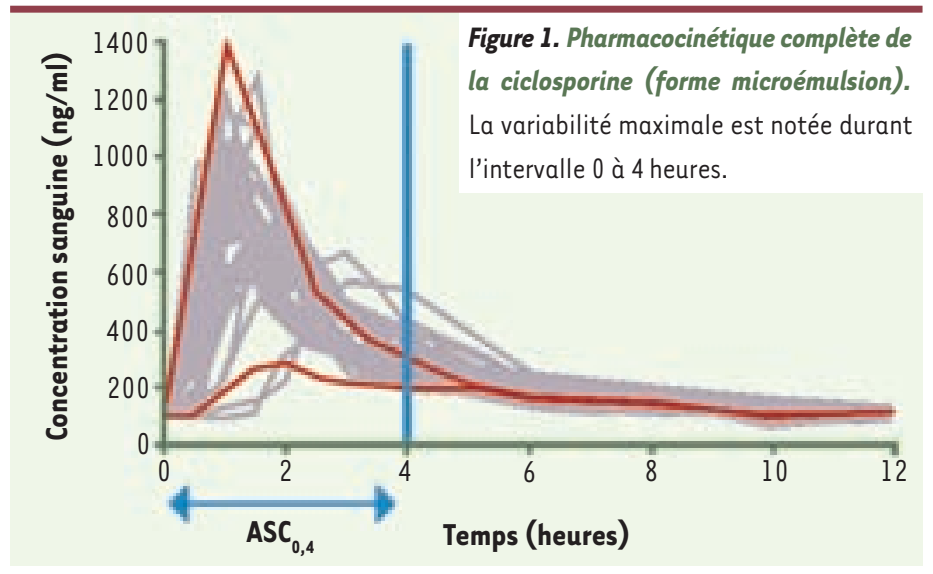

Les résultats chez les patients en situation stable et après la première année suivant la transplantation sont encore plus rares. Une seule étude portant sur 70 patients a été réalisée pour comparer après trois mois un suivi par la C2 et un suivi par la CO [14]. Les incidences de rejet aigu et de néphrotoxicité étaient identiques dans les deux groupes. $\varepsilon$ n revanche, l'utilisation de la C2 était associée à une diminution d'environ $20 \%$ de la posologie quotidienne de CsA sans augmentation de l'incidence de rejet aigu, mais à une diminution de la posologie des traitements antihypertenseurs. En conclusion, même si il existe des arguments théoriques pour la supériorité du suivi par la C2, il n'existe pas de preuve de sa supériorité en clinique.

Compte tenu des limites du suivi pharmacologique actuel, d'organe. Ce lien persiste quels que soient les traitements (en particulier avec une induction biologique, c'est-àdire l'utilisation d'un traitement par des anticorps polyclonaux ou monoclonaux dirigés contre les lymphocytes T) utilisés à la période initiale après la transplantation. De plus, l'obtention de concentrations adéquates dès 5 jours après transplantation est importante pour obtenir la plus faible incidence de rejet. L'utilisation de la C2 est également intéressante car elle permet d'individualiser une population particulière présentant des C2 faibles malgré des posologies quotidiennes élevées. Dans ce cas, la réalisation d'un dosage plus tardif, par exemple 6 heures après la prise (C6), ou une AUC complète, permet de différencier les véritables mauvais absorbeurs, qui ont une $\mathrm{C} 6$ basse, des patients présentant un retard à l'absorption ( 6 > C2), comme c'est le cas en présence d'une gastroparésie diabétique. Dans les périodes plus tardives suivant la transplantation, l'utilisation de la C2 pourrait diminuer les complications liées à la CsA, comme la néphrotoxicité, l'hypertrophie gingivale ou l'hirsutisme. Cette conférence de consensus a également donné lieu à la définition de valeurs cibles selon le type d'organe et la période après la transplantation qui ont été précisées lors d'une conférence de consensus plus récente (Tableau I) [12]. Cette conférence a aussi mis en avant quelques limitations de cette stratégie en indiquant que d'autres outils (comme des AUC courtes) sont parfois utiles.

D'autres critiques ont été avancées plus récemment. Knight et Morris ont conduit une revue systématique des études portant sur le bénéfice clinique d'un suivi par la C2 [13]. Les auteurs ont identifié 29 études, dont 10 avaient une méthodologie adéquate (contrôlée et randomisée). Dans la période initiale suivant la transplantation, I'utilisation de la C2 par rapport à la CO est associée à une augmentation de $50 \%$ en moyenne de la dose de CsA utilisée. Elle n'était pas associée à une diminution significative de l'incidence de rejet aigu, mais ne s'accompagnait pas non plus d'une dégradation significative de la fonction rénale. en particulier à distance de la transplantation, de nouvelles modalités sont proposées. II peut s'agir d'AUC abrégées, utilisant des outils bayésiens. Cette approche a été utilisée par l'équipe de Néphrologie de Rouen. Lors d'une étude prospective, cette équipe a montré que l'adaptation de la dose quotidienne de CsA pour obtenir une cible basse pour l'AUC abrégée $(2,0$ à 2,6 $\mu \mathrm{g}$.h/l) chez des patients après la première année de greffe améliore significativement la fonction rénale sans augmentation du risque de rejet aigu (Etienne I, communication personnelle).

\section{Exemple 2 : inhibiteur de l'inosine monophosphate déshydrogénase: Acide mycophénolique et dérivés}

Les inhibiteurs de l'IMPDH sont très utilisés dans les stratégies immunosuppressives après transplantation. Les deux traitements actuellement disponibles dans ce cadre sont le mycophénolate mofétil (MMF) et le mycophénolate de sodium (MPS). Une question récente est celle de l'intérêt clinique de réaliser un STP.

Le MMF est le morpholinoethylester du MPA, qui est le principe actif. II est rapidement hydrolysé dans le tube digestif supérieur, pour produire du MPA [15]. Le MPS libère le MPA à un pH neutre dans l'intestin grêle avec de ce fait une absorption plus lente [16]. Le MPA est métabolisé principalement dans le foie par les enzymes du système uridine diphosphonate glucuronyltransférase (UGT). La comparaison du MMF et du MPS montre que ce dernier présente une concentration maximale (Cmax) retardée en relation avec une absorption plus tardive [17]. En revanche, même en normalisant les résultats en fonction de la dose utilisée, c'est-à-dire en divisant le résultat par la posologie quotidienne, la Cmax et l'AUC obtenue avec le MPS sont plus élevées qu'avec le MMF [18].

Dans tous les cas, le STP ne peut être fait que par le dosage du MPA. Plusieurs approches ont été tentées pour réaliser le STP du médicament. La première approche consiste à doser le CO du MPA, mais les résultats sont sujets à caution puisque l'existence d'un deuxième pic plasmatique entraîne des modifications non reproductibles fonction de nombreux facteurs en particulier alimentaires. La seconde approche consiste à étudier l'aire sous la courbe du MPA. II existe différentes méthodes pour calculer l'aire sous la courbe du MPA. La plus fiable consiste à réaliser de multiples prélèvements sanguins pour calculer ainsi une AUC complète. 
Cette méthode est inutilisable en pratique clinique car elle représente trop de contraintes pour le patient et le personnel soignant. Une autre méthode consiste à réaliser une stratégie de prélèvements limités à deux ou trois temps après la prise [19]. Des formules mathématiques permettent de calculer l'AUC. L'utilisation d'algorithmes de régression linéaire a l'inconvénient majeur de nécessiter un strict respect des heures de prélèvements. L'estimation bayésienne, méthode mathématique et statistique, permet, à partir de 3 prélèvements sanguins, de calculer les AUC et présente l'avantage d'une plus grande souplesse pour les heures de prélèvements [20]. Les estimateurs bayésiens permettent le calcul de I'AUC et l'adaptation de posologie pour fixer la nouvelle dose en fonction de la cible thérapeutique ${ }^{1}$.

La question se pose de l'intérêt clinique d'utiliser le STP dans le cas du MMF. Une étude dans laquelle les concentrations étaient contrôlées a conclu que les patients du groupe à faible exposition présentent une incidence élevée de rejet [21]. Pour les autres études, le taux de rejet était sensiblement identique, avec une incidence plus élevée d'effets secondaires dans le groupe cible avec la valeur la plus haute. Dans le cas du MPA, les conférences de consensus recommandent l'utilisation du STP et proposent une cible thérapeutique comprise entre 30 et $60 \mathrm{mg}$ / $\mathrm{h} / \mathrm{I}[15,19]$. Plus récemment, deux études prospectives randomisées explorant l'intérêt du STP ont apporté des résultats contradictoires. La première étude internationale, non encore publiée, n'a pas montré d'amélioration de l'incidence de rejet aigu ou des effets indésirables en cas de STP (T. Van Gelder, communication personnelle). Cette étude est entachée de nombreux biais méthodologiques, le plus important étant que les adaptations posologiques n'ont pas été réalisées. La deuxième étude française a étudié la valeur du STP dans une population homogène de patients, avec une adaptation réalisée effectivement selon I'AUC calculée par méthode bayésienne et des concentrations obtenues par HPLC [22]. Dans ce contexte, le STP réalisé à J7, J14, M3 et M6 pour obtenir une AUC cible de $40 \mathrm{mg} / \mathrm{h} / \mathrm{l}$, est associé à moins d'échecs de traitement et de rejet aigu sans augmentation des événements indésirables. En raison du caractère contradictoire des deux études cliniques citées, le bénéfice d'un STP systématique pour le MMF est discutable : il peut être proposé à la période initiale de la transplantation et en cas d'évolution clinique inhabituelle.

${ }^{1}$ Le terme cible thérapeutique est l'expression consacrée pour définir la zone thérapeutique de concentration sanguine qu'il est souhaitable d'obtenir.
Pour le MPS, en raison d'une variabilité plus marquée des caractéristiques pharmacocinétiques en relation avec une absorption moins prévisible, il n'a pas été possible pour l'instant de proposer une approche de I'AUC par des algorithmes de régression linéaire ou des estimateurs bayésiens.

\section{Exemple 3 : le Tacrolimus}

Une conférence de consensus non encore publiée, a fait un point sur les connaissances actuelles portant sur le TAC et a proposé des cibles thérapeutiques (Tableau II) (P. Wallemacq, communication personnelle). Une étude récente en transplantation rénale a montré que des $\mathrm{CO}$ cibles comprises entre 3 et $7 \mathrm{ng} / \mathrm{ml}$ étaient associés aux meilleurs résultats [23]. Dans cette étude, les patients recevant du TAC présentaient une meilleure fonction rénale, une moindre incidence de rejet aigu et une meilleure survie à un an du greffon rénal par comparaison avec les groupes traités avec de fortes ou de faibles doses de CsA ou avec du sirolimus combinés aux autres traitements (MMF et stéroïdes).

L'actualité du TAC en pharmacologie porte sur l'intérêt de la pharmacogénétique pour obtenir des concentrations souhaitées, appelées aussi concentrations cibles ou targeted concentrations, plus précocement et de façon plus reproductible. La principale enzyme impliquée dans le métabolisme du TAC est la sous-famille 3A du cytochrome P450 [24]. Les activités CYP3A chez l'homme reflètent l'expression hétérogène d'au moins deux isoformes de cette sous-famillle, les CyP3A4 et CyP3A5, dont les gènes sont localisés de façon adjacente sur le chromosome 7q21. En ce qui concerne le gène CyP3A4, plus de 20 SNP (single nucleotide polymorphisms) ont été décrits. Une étude portant sur un SNP localisé dans la région 5' du gène, responsable de l'allèle CYP3A4* $1 B$, a montré que les patients porteurs de cet allèle nécessitent une dose plus faible de TAC pour obtenir la concentration cible [25]. Il est admis cependant que l'isoforme CYP3A5 est la plus importante pour le métabolisme du TAC.

\begin{tabular}{|c|c|c|c|c|c|}
\hline $\begin{array}{l}\text { Délai } \\
\text { (mois) }\end{array}$ & $\begin{array}{l}\text { Sans } \\
\text { induction }\end{array}$ & AcMo antiCD25 & Ac polyclonaux/HRI & $\begin{array}{l}\text { MMF/minimisation } \\
\text { des stéroïdes }\end{array}$ & Inh mTOR \\
\hline \multicolumn{6}{|c|}{ Trithérapie } \\
\hline $0-3$ & $10-15$ & $3-7$ & $5-10 / 10-15$ & $10-15$ & $3-7$ \\
\hline $3-12$ & $5-15$ & $3-7$ & $5-10 / 10-15$ & $8-12$ & $3-7$ \\
\hline$>12$ & $5-10$ & $3-7$ & $5-10 / 8-12$ & $5-10$ & $3-7$ \\
\hline
\end{tabular}

Tableau II. Recommandations pour les concentrations cibles (CO) de tacrolimus en ng/ml après transplantation. AntiCD25: anticorps monoclonal anti-récepteur de l'interleukine-2; HRI : haut risque immunologique; MMF : mycophénolate mofétil ; Inh mTOR: inhibiteurs de mammalian target of rapamycine (P. Wallemacq, communication personnelle). 
Seuls les individus avec au moins un allèle CYP3A5* 1 (A à la position 6986) produisent des taux élevés d'ARNm et expriment la protéine CYP3A5, qui représente alors au moins $50 \%$ du contenu total en CyP3A [26]. Les porteurs de l'allèle CyP3A5*3 ( $G$ en position 6986 ) ont une variabilité de séquence dans l'intron 3 qui crée un site d'épissage et code pour un ARNm tronqué avec un codon stop prématuré. Nous avons montré qu'il existe une association entre les besoins en TAC et ce polymorphisme (Figure 2) [27]. Les patients avec un génotype CYP3A5* $1 /{ }^{*}$ ont un métabolisme intestinal et hépatique important, et la dose quotidienne nécessaire pour obtenir des taux résiduels de TAC adéquats est plus élevée. La présence d'un allèle ${ }^{\star} l$ est associée à la survenue plus précoce des épisodes de rejet après transplantation en raison d'une concentration inadéquate dans le contexte d'un traitement initial sans induction biologique [28]. La plus grande fréquence de cet allèle dans la population noire pourrait expliquer en partie les moins bons résultats observés après transplantation rénale dans cette population. En utilisant le génotypage de patients inscrits sur la liste d'attente pour lesquels une pharmacocinétique complète de TAC était réalisée, des posologies initiales adaptées au génotype ont été déterminées [29]. Elles seraient de $0,30 \mathrm{mg} / \mathrm{kg} / \mathrm{j}$ pour les patients exprimant le CyP3A5 (allèle * 1 ) et de $0,15 \mathrm{mg} / \mathrm{kg} / \mathrm{j}$ pour les patients ne l'exprimant pas $(* 3 / * 3)$. Nous venons d'achever une étude multicentrique randomisée comparant des patients pour lesquels la posologie initiale était, soit celle préconisée par l'autorisation de mise sur le marché (AMM), soit adaptée au génotype. Les résultats devraient permettre de préciser l'intérêt clinique d'une adaptation pharmacogénétique de ce traitement.

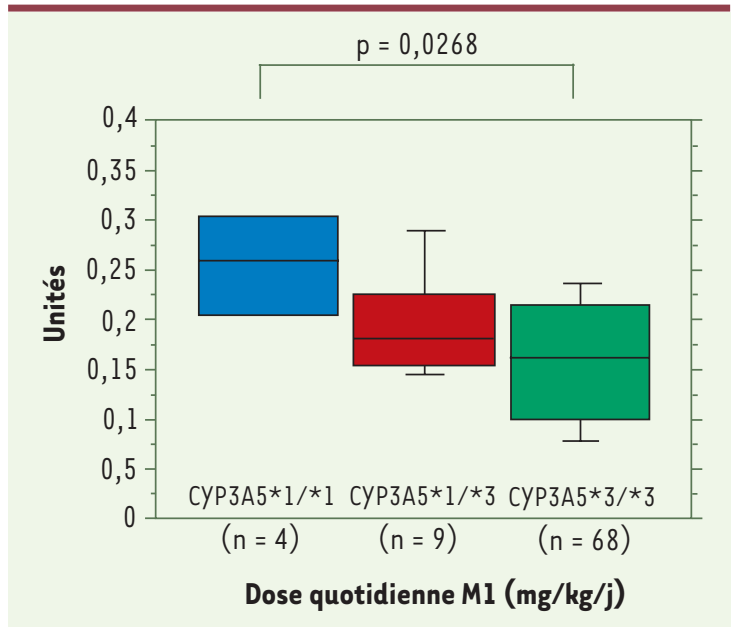

Figure 2. Dose de tacrolimus utilisée un mois après la transplantation en fonction du génotype des patients pour le cytochrome P450 3 A5.

\section{Exemple 4 : Sirolimus}

Une nouvelle classe de traitements immunosuppresseurs est représentée par les inhibiteurs de la mammalian target of rapamycin (mTOR). En raison de ses effets antiprolifératifs, cette famille mTOR pourrait être particulièrement intéressante lorsqu'il existe une dysfonction chronique du greffon ou une complication tumorale après transplantation. $\varepsilon$ n raison d'une augmentation de la néphrotoxicité des inhibiteurs de la calcineurine en cas d'utilisation concomitante avec les inhibiteurs de mTOR, le sirolimus est utilisé sans CsA ni TAC dès la période initiale suivant la transplantation. Dans ce cas, il faut obtenir rapidement les concentrations résiduelles souhaitées pour éviter les rejets aigus, sans dépasser la cible en raison des complications précoces (reprise retardée de fonction ou cicatrisation). L'obtention de ces cibles est souvent difficile. Une piste pour améliorer ces difficultés est l'utilisation de la pharmacogénétique. Nous avons montré avec d'autres que les doses de sirolimus nécessaires pour obtenir les concentrations résiduelles adéquates sont corrélées avec le polymorphisme du CYP3A5 [30, 31$]$. Une étude prospective randomisée doit débuter pour démontrer l'intérêt clinique de cette approche.

\section{Conclusion}

Les nouveaux outils de la pharmacologie ont permis d'améliorer l'individualisation du traitement immunosuppresseur. Une autre dimension que nous n'avons pas évoquée ici est la possibilité d'utiliser l'immunopharmacogénétique. Dans ce cadre, la détermination de polymorphismes génétiques ou d'une signature transcriptomique spécifique d'une population opérationnellement tolérante pourrait permettre d'adapter individuellement le type de traitement et de proposer une minimisation voire un arrêt complet des traitements chez certains patients [32]. $\diamond$

\section{SUMMARY}

Recents advances in the pharmacology of immunosuppressive drugs Individualization of immunosuppressive drugs may be helped by the use of therapeutic drug monitoring determining the best unique time point for concentration to be controlled or using limited sample strategy to perform abbreviated area under the curve (AUC). Pharmacogenetic analyzes the role of enzymes genetic polymorphisms on pharmacokinetic characteristics. Recent studies have demonstrated that cyclosporine concentration obtained 2 hours after oral intake is the best single point. However, a recent analysis of published data did not sustain the clinical benefit of this approach. Regarding mycophenolate mofetil, the targeted AUC has been defined between 30 and $60 \mathrm{mg} / \mathrm{h} / \mathrm{l}$. Clinical results are contradictory and it is not possible for the time being to give a clear answer to its clinical relevance. For tacrolimus, it has been demonstrated that the daily dose needed to achieve adequate trough levels are correlated with cytochrome P450 3A5 expression and therefore the presence of ${ }^{*} 1$ allele. A prospective study has been conducted to demonstrate the clinical consequences of this pharmacogenetic approach. $\diamond$ 


\section{RÉFÉRENCES}

1. Myers BD, Ross J, Newton L, et al. Cyclosporine-associated chronic nephropathy. N Engl J Med 1984 ; $311: 699-705$.

2. Lindholm A, Kahan BD. Influence of cyclosporine pharmacokinetics, trough concentrations, and AUC monitoring on outcome after kidney transplantation. Clin Pharmacol Ther 1993 ; 54 : 205-18.

3. Kasiske BL, Heim-Duthoy K, Rao KV, Awni WM. The relationship between cyclosporine pharmacokinetic parameters and subsequent acute rejection in renal transplant recipients. Transplantation $1988 ; 46: 716-22$.

4. Keown $P$, Landsberg $D$, Halloran $P$, et al. A randomized, prospective multicenter pharmacoepidemiologic study of cyclosporine microemulsion in stable renal graft recipients. Report of the Canadian neoral renal transplantation study group. Transplantation $1996 ; 62$ : 1744-52.

5. Barone G, Chang CT, Choc MG Jr, et al. The pharmacokinetics of a microemulsion formulation of cyclosporine in primary renal allograft recipients. The neoral study Group. Transplantation 1996 ; $61: 875-80$.

6. Mahalati K, Belitsky P, Sketris I, et al. Neoral monitoring by simplified sparse sampling area under the concentration-time curve: its relationship to acute rejection and cyclosporine nephrotoxicity early after kidney transplantation. Transplantation $1999 ; 68: 55-62$.

7. International neoral renal transplantation study group. Cyclosporine microemulsion (Neoral) absorption profiling and sparse-sample predictors during the first 3 months after renal transplantation. Am J Transplant $2002 ; 2: 148-56$.

8. Cantarovich M, Barkun JS, Tchervenkov JI, et al. Comparison of neoral dose monitoring with cyclosporine through levels versus 2 -hr postdose levels in stable liver transplant patients. Transplantation 1998; $66: 1621-7$.

9. Cantarovich M, Besner JG, Barkun JS, et al. Two-hour cyclosporine level determination is the appropriate tool to monitor Neoral therapy. Clin Transplant $1998 ; 12: 243-9$.

10. Jaksch P, Kocher A, Neuhauser P, et al. Monitoring C2 level predicts exposure in maintenance lung transplant patients receiving the microemulsion formulation of cyclosporine (Neoral). J Heart Lung Transplant $2005 ; 24: 1076-80$

11. Levy G, Thervet $\varepsilon$, Lake J, Uchida K. Patient management by Neoral C(2) monitoring: an international consensus statement. Transplantation $2002 ; 73$ (suppl 9) : S12.

12. Nashan B, Bock A, Bosmans JL, et al. Use of Neoral C monitoring: a European consensus. Transpl Int $2005 ; 18: 768-78$.

13. Knight SR, Morris PJ. The clinical benefits of cyclosporine C2-level monitoring: a systematic review. Transplantation $2007 ; 83: 1525-35$.

14. Glanville AR, Aboyoun CL, Morton JM, et al. Cyclosporine C2 target levels and acute cellular rejection after lung transplantation. J Heart Lung Transplant 2006 ; 25 : 928-34.

15. Shaw LM, Figurski M, Milone MC, et al. Therapeutic drug monitoring of mycophenolic acid. Clin J Am Soc Nephrol 2007 ; 2 : 1062-72.

16. Gabardi S, Tran JL, Clarkson MR. Enteric-coated mycophenolate sodium. Ann Pharmacother 2003; $37: 1685-93$.

17. Budde K, Glander P, Diekmann F, et al. Review of the immunosuppressant enteric-coated mycophenolate sodium. Expert Opin Pharmacother 2004 ; $5: 1333-45$.

18. Tedesco-Silva H, Bastien MC, Choi L, et al. Mycophenolic acid metabolite profile in renal transplant patients receiving enteric-coated mycophenolate sodium or mycophenolate mofetil. Transplant Proc $2005 ; 37: 852-5$.

19. Van Gelder T, Le Meur Y, Shaw LM, et al. Therapeutic drug monitoring of mycophenolate mofetil in transplantation. Ther Drug Monit $2006 ; 28$ : 145-54.
20. Premaud A, Debord J, Rousseau A, et al. A double absorption-phase model adequately describes mycophenolic acid plasma profiles in de novo renal transplant recipients given oral mycophenolate mofetil. Clin Pharmacokinet $2005 ; 44: 837-47$.

21. Van Gelder T, Hilbrands LB, Vanrenterghem Y, et al. A randomized double-blind, multicenter plasma concentration controlled study of the safety and efficacy of oral mycophenolate mofetil for the prevention of acute rejection after kidney transplantation. Transplantation $1999 ; 68: 261-6$.

22. Le Meur $Y$, Buchler M, Thierry A, et al. Individualized mycophenolate mofetil dosing based on drug exposure significantly improves patient outcomes after renal transplantation. Am J Transplant $2007 ; 7: 2496-503$.

23. Ekberg H, Tedesco-Silva H, Demirbas A, et al. Reduced exposure to calcineurin inhibitors in renal transplantation. $N$ Engl J Med 2007 ; 357 : 2562-75.

24. Thervet $\varepsilon$, Legendre C, Beaune P, Anglicheau D. Cytochrome P450 $3 \mathrm{~A}$ polymorphisms and immunosuppressive drugs. Pharmacogenomics 2005 ; $6: 37-47$.

25. Hesselink DA, van Schaik RH, van der Heiden IP, et al. Genetic polymorphisms of the CYP3A4, CYP3A5, and MDR-1 genes and pharmacokinetics of the calcineurin inhibitors cyclosporine and tacrolimus. Clin Pharmacol Ther 2003; $74: 245-54$.

26. Kuehl P, Zhang J, Lin Y, et al. Sequence diversity in CYP3A promoters and characterization of the genetic basis of polymorphic CYP3A5 expression. Nat Genet $2001 ; 27: 383-91$.

27. Thervet $\varepsilon$, Anglicheau D, King B, et al. Impact of cytochrome p450 3 A5 genetic polymorphism on tacrolimus doses and concentration-to-dose ratio in renal transplant recipients. Transplantation $2003 ; 76: 1233-5$.

28. MacPhee IA, Fredericks S, Tai T, et al. The influence of pharmacogenetics on the time to achieve target tacrolimus concentrations after kidney transplantation. Am J Transplant $2004 ; 4$ : 914-9.

29. Haufroid V, Wallemacq P, VanKerckhove V, et al. CYP3A5 and ABCB 1 polymorphisms and tacrolimus pharmacokinetics in renal transplant candidates: guidelines from an experimental study. Am J Transplant 2006 ; $6: 2706-13$.

30. Le Meur Y, Djebli N, Szelag JC, et al. CYP3A5*3 influences sirolimus oral clearance in de novo and stable renal transplant recipients. Clin Pharmacol Ther $2006 ; 80: 51-60$.

31. Anglicheau D, Le Corre D, Lechaton S, et al. Consequences of genetic polymorphisms for sirolimus requirements after renal transplant in patients on primary sirolimus therapy. Am J Transplant $2005 ; 5: 595-603$.

32. Brouard S, Mansfield $\varepsilon$, Braud C, et al. Identification of a peripheral blood transcriptional biomarker panel associated with operational renal allograft tolerance. Proc Natl Acad Sci USA 2007 ; 104 : 15448-53.

33. Pallet $N$, Beaune $P$, Thervet $\varepsilon$, et al. Inhibiteurs de $m T O R$ : des antiprolifératifs pléiotropiques. Med Sci (Paris) 2006 ; 22 : 947-52.

\section{TIRÉS À PART}

\section{$\varepsilon$. Thervet}

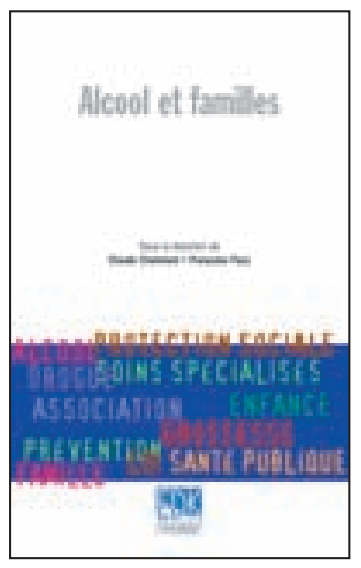

ISBN : 978-2-8425-4116-3 174 pages

\section{Bon de commande}

À retourner à EDK, 2, rue Troyon - 92316 Sèvres Cedex

Tél. : 0155641393 - Fax : 0155641394 - E-mail : edk@edk.fr

NOM : Prénom :

Adresse :

Code postal : Ville :

Pays :

Fonction :

Je souhaite recevoir l'ouvrage Alcool et familles : $15 €+3 €$ de port $=\mathbf{1 8} €$ TTC

en ................. exemplaire, soit un total de $€$

$\square$ Par chèque, à l'ordre de $\mathbf{E} \mathbf{D} \mathbf{K}$

$\square$ Par carte bancaire :

Carte $n^{\circ}$

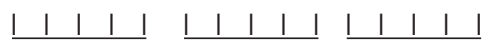

Date d'expiration :

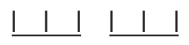

$\mathrm{N}^{\circ}$ de contrôle au dos de la carte : 